\title{
Performance Effects of Repetition Specific Gluteal Activation Protocols on Acceleration in Male Rugby Union Players
}

\author{
by \\ Lorna Barry ${ }^{1,2}$, Ian Kenny ${ }^{1}$,Thomas Comyns ${ }^{1}$
}

\begin{abstract}
Warm-up protocols have the potential to cause an acute enhancement of dynamic sprinting performance. The purpose of this study was to evaluate the effects of three repetition specific gluteal activation warm-up protocols on acceleration performance in male rugby union players. Forty male academy rugby union players were randomly assigned to one of 4 groups (control, 5, 10 or 15 repetition gluteal activation group) and performed $10 \mathrm{~m}$ sprints at baseline and 30 s, 2, 4, 6 and 8 min after their specific intervention protocol. Five and ten meter sprint times were the dependent variable and dual-beam timing gates were used to record all sprint times. Repeated measures analysis of variance found no significant improvement in 5 and $10 \mathrm{~m}$ sprint times between baseline and post warm-up scores ( $p \geq$ 0.05) for all groups. There were no reported significant differences between groups at any of the rest interval time points $(p \geq 0.05)$. However, when individual responses to the warm-up protocols were analyzed, the 15 repetition gluteal activation group had faster $10 \mathrm{~m}$ times post-intervention and this improvement was significant $(p=0.021)$. These results would indicate that there is no specific rest interval for any of the gluteal interventions that results in a potentiation effect on acceleration performance. However, the individual response analysis would seem to indicate that a 15 repetition gluteal activation warm-up protocol has a potentiating effect on acceleration performance provided that the rest interval is adequately and individually determined.
\end{abstract}

Key words: plyometrics, rest interval, sprinting, warm-up.

\section{Introduction}

The development of professional rugby in 1995 has led to a greater input of sport science support in the game. More emphasis has been placed on high performance practices in rugby with a particular focus on a pre-performance warm-up and improving athlete's power production as well as speed capability. Baker and Nance (1999) identified that speed and acceleration were important qualities in field sports, and an enhanced ability to sprint over short distances was fundamental to success. The warm-up that is performed before sprinting can have both a potentiating and fatiguing effect on the performance of this dynamic activity.
Research has been undertaken to assess the postactivation potentiation (PAP) effect of warm-up protocols on sprinting performance (Bevan et al., 2010; Chatzopoulos et al., 2007; Comyns et al., 2010; McBride et al., 2005; Turner et al., 2015). Such studies have used varying methods to induce a PAP effect on sprinting, for example heavy lifting and plyometrics.

Since its conception, PAP has gained substantial interest both as a means of developing power and as a warm-up tool (Matthews et al., 2010). The goal of such a warm-up is to generate a PAP effect which has been described as an acute enhancement of muscle function after a preload

1 - Biomechanics Research Unit, University of Limerick, Limerick, Ireland.

2 - Sports Santry Clinic, Demesne, Dublin 9, Ireland. 
stimulus (Bevan et al., 2010). Practically, warmups for explosive activities have attempted to elicit a PAP effect by coupling a heavy load exercise with an explosive movement (Hodgson et al., 2005). However, fatigue can be induced during this process which has been shown to have a negative effect on sprinting performance (Comyns et al., 2010). Previous research has explored the effectiveness of heavy pre-load muscle stimulation on sprinting, but with conflicting results due to the study designs incorporating varying pre-load stimulus protocols and rest intervals (Bevan et al., 2010; Chatzopoulos et al., 2007; Comyns et al., 2010; McBride et al., 2005; Turner et al., 2015 ). McBride et al. (2005) advocated the use of a $90 \%$ of 1 RM back squat for three repetitions as being capable of improving 40 m sprint times. In contrast, Comyns et al. (2010) observed no significant changes in $30 \mathrm{~m}$ sprint times over four sessions when a 3RM back squat was applied. Chatzopoulos et al. (2007) demonstrated that $10 \mathrm{~m}$ and $30 \mathrm{~m}$ velocity was unchanged 3 minutes after the completion of ten single repetitions at $90 \%$ of $1 \mathrm{RM}$. It is thought that fatigue was induced by the warm-up protocol and further research into a longer rest interval is necessary (Chatzopoulos, 2007). Bevan et al. (2010) explored the effect of a back squat protocol on $10 \mathrm{~m}$ sprinting speed in 16 male rugby union players. Initial results showed no significant time effect with regard to $5 \mathrm{~m}$ and $10 \mathrm{~m}$ sprint times after 1 set of 3 repetition back squats at $91 \%$ of $1 \mathrm{RM}$. However, when individual responses to the stimulus were taken into account a significant improvement in sprint performance was observed compared with the individuals' baseline sprint. Bevan et al. (2010) concluded that sprinting performance was enhanced after a heavy preload stimulus providing adequate and individualized recovery was applied.

The equivocal nature of these studies is said to be due in part to variability within the methodologies and research design. Factors including the type of preload stimulus, rest interval duration and the type of explosive activity are often not uniform among different studies causing discrepancies in the results and hindering comparisons (Hodgson et al., 2005). On a practical note, problems have also arisen when using heavy loads ( $>80 \%$ of $1 \mathrm{RM}$ ) as warm-up routines. Athletes, for example, may not have sufficient technical capability to safely or effectively execute a movement pattern under such a heavy load. While the acute nature of the muscle activation means access to weight lifting equipment on site immediately prior to competition is necessary, it is not practical in most sporting events. To overcome such issues recent research has examined the PAP effect of gluteal activation exercises on dynamic performance (Comyns et al., 2015; Crow et al., 2012; Healy and Harrison, 2014). The gluteal muscle groups have been the focus of such research as they have been deemed the most important muscles for forward propulsion in sprinting (Novacheck, 1998). The gluteal muscles play a major role in running and jumping activities, and studies researching lower limb activities during running and jumping have found the gluteal muscles to be vigorously activated (Kyrolainen et al., 2005; Nagano et al., 2005; Palastanga et al., 2002). Wiemann and Tidow (1995) suggested that during the initial support phase of sprinting the medial gluteal muscle acted to prevent the dropping of the pelvis at the opposite side and thus, together with the oblique muscles, stabilised the pelvis and the trunk, allowing for a more effective and quicker sprinting action.

Crow et al. (2012) researched the acute effect of low load gluteal exercises on power output. These researchers hypothesized that, unlike heavy load protocols, a low intensity preload would improve explosive power output in elite athletes without inducing fatigue. Upon completion of 1 set of 10 repetitions of seven gluteal specific exercises and a five minute recovery, 5 consecutive countermovement jumps (CMJ) were recorded. Peak power output in the $\mathrm{CMJ}$ was found to be significantly higher following the performance of the gluteal warm-up protocol. Despite the CMJ height not being recorded, the findings suggest that a low-load gluteal warm-up may be effective in enhancing explosive jump performance. Comyns et al. (2015) also found that a similar low load gluteal warmup was capable of augmenting force production variables in a squat jump provided an 8 minute rest period was implemented between the gluteal warm-up protocol and subsequent jump performance. Neither study was able to suggest that low load gluteal activation was able to improve performance outcome measures such as 
jump height; nevertheless those participating in sports with an explosive force production element may find it beneficial. Healy and Harrison (2014) looked at the effectiveness of a similar low load gluteal warm-up on single leg drop jump performance. Fifteen sprint trained track athletes completed three separate testing days. Days one and three acted as a control day and consisted of a standardized warm-up followed by 10 single leg drop jumps with one minute intervals. Day two acted as the intervention day and consisted of a standardized warm-up, the gluteal activation protocol, followed by 10 single leg drop jumps. Initial results suggested that significant differences found between the mean scores between days 1 and 2 could have been as a result of the gluteal intervention. However, further analysis did not show similar significant differences between day 2 and day 3. Healy and Harrison (2014) suggested that initial improvements were a result of the learning effect and not the gluteal intervention. While the above studies have presented findings both for and against the use of a low load gluteal specific warm-up, it highlights the need for specificity in exercise selection, recovery intervals and an individual program design when implementing a warm-up utilizing a gluteal activation protocol.

Building on this research, the present study sought to research the effect of a low load gluteal warm-up on sprinting performance in male rugby union players. The intention was to research the effect on acceleration performance of various repetition specific gluteal activation warm-up protocols that could be effectively incorporated into a pre-competition routine in rugby union. It is widely accepted that speed, and in particular acceleration, are potentially decisive factors in successful outcomes in rugby competitions. Therefore, researching the PAP effects on acceleration of a low intensity pre-load protocol that required little equipment to complete and could be incorporated into a precompetition warm-up routine would be highly beneficial to rugby players. Logistically it has the potential to provide a user-friendly method of inducing a PAP effect pre-competition that could enhance players' match performance. The study also aimed to identify the rest interval that would result in the most significant improvement, if any, in acceleration performance.

\section{Material and Methods}

\section{Participants}

Forty male academy level rugby union players formed the subject base for this study (age: $18.25 \pm 2.5$ years; body height: $181.25 \pm 6.55$ $\mathrm{cm}$; body mass: $79.7 \pm 11.3 \mathrm{~kg}$ ). Participants had a minimum of 2 years of structured strength and conditioning training and had previous experience with gluteal activation exercises and acceleration. The participants were injury-free and were in their pre-season period at the time of the study. Approval from the University of Limerick Ethics Committee was received prior to participant recruitment. Participants were informed of the experimental risks and signed an informed consent form and completed a Physical Activity Readiness Questionnaire before the commencement of the study.

\section{Procedures}

The study involved each participant attending two separate testing sessions over a two-week period. For reliability reasons and to control for circadian variation, each participant completed all sessions on the same day of the week and at the same time (Atkinson and Reilly, 1996). Prior to the start of the testing sessions, the participants were randomly assigned to one of four groups, which were the control, 5-repetition (5R), 10-repetition (10R), and 15-repetition (15R) group. Testing session one was a familiarizaton session where the participants were instructed on the technique of the gluteal exercises. The second testing session was the intervention session.

Prior to the start of testing session one, the participants undertook a warm-up procedure consisting of low-intensity aerobic exercises that involved jogging and skipping, followed by dynamic stretching of each of the major muscles groups in the lower body. Subsequently, the participants were familarized with the starting position for the $10 \mathrm{~m}$ sprints that were assessed in the second testing session. The 5R, 10R and 15R groups were also instructed on the correct technique for each of the seven exercises of the gluteal intervention. These exercises replicated the low-load gluteal exercises employed by Comyns et al. (2015), Crow et al. (2012) and Healy and Harrison (2014). The exercises were a double leg bridge, quadruped lower extremity lift, quadruped hip abduction, side lying clams in $60^{\circ}$ hip flexion, side lying hip abduction, prone single 
leg hip extension and stability ball wall squats (Table 1). Each movement was held for one second before returning to the starting position and progressing to the next repetition. Fifteen second rest was allowed between each exercise.

On the second testing day the participants completed a movement specific warm-up in order to prepare the athlete for the maximal effort baseline trials (Jeffreys, 2006; Little and Williams 2006). The warm-up included a light jog lasting for approximately three minutes, a controlled dynamic stretch of all major lower body muscles and a sequence of four submaximal accelerations over $10 \mathrm{~m}$ at $75 \%, 85 \%, 90 \%$ and $100 \%$ self-selected efforts. The recovery period between each sub-maximal acceleration run was approximately 90 seconds. After the warm-up, the participants completed three baseline $10 \mathrm{~m}$ trials at $100 \%$ effort with a rest interval of $90 \mathrm{~s}$ between each trial. The participants then performed their group specific intervention. The $5 \mathrm{R}, 10 \mathrm{R}$ and $15 \mathrm{R}$ groups completed the same seven exercises that are detailed in Table 1 . The $5 R$ group performed 5 repetitions of each exercise, the $10 \mathrm{R}$ group did 10 repetitions and finally the $15 R$ group executed 15 repetitions of each exercise. The control group had a 10 min rest period at this time that mirrored on average the duration of the gluteal interventions.

After each group completed their specific intervention, the participants completed five post sprint $10 \mathrm{~m}$ trials through timing gates at predetermined rest intervals $(30 \mathrm{~s}, 2 \mathrm{~min}, 4 \mathrm{~min}, 6$ $\mathrm{min}$ and $8 \mathrm{~min})$. Participants were required to complete a cool down consisting of light jogging and static stretching of each of the major lower body muscle groups.

\section{Instrumentation}

All $10 \mathrm{~m}$ sprints were performed in a sports hall with ample space to conduct both the $10 \mathrm{~m}$ sprint assessment and the gluteal warm-up protocol. Sprint times over 5 and $10 \mathrm{~m}$ were recorded using Racetime 2, dual-beam timing gates (Microgate, Botzano, Italy). The timing gates were positioned at the $0 \mathrm{~m}, 5 \mathrm{~m}$ and $10 \mathrm{~m}$ mark and set at a height of $0.8 \mathrm{~m}$ using methods similar to Whelan et al. (2014). Participants were required to start each sprint from a standard two point (standing) starting position with their front foot placed behind a line $0.7 \mathrm{~m}$ from the first set of timing gates thus ensuring that they did not trigger the timing gates before the start of each sprint. Dual beam timing gates are considered reliable as a means of testing $10 \mathrm{~m}$ sprinting performance for they eliminate the occurrence of false signals when compared to single beam timing gates (Earp and Newton, 2012). The three baseline trials for all participants were used to confirm the reliability of the testing equipment and protocols. The reported ICC for $10 \mathrm{~m}$ time was 0.955 and the coefficient of variation $(\mathrm{CV})$ was $1.4 \%$. Research suggests that a test is only deemed reliable if the $\mathrm{CV} \leq 10 \%$ and the ICC $\geq$ 0.80 (Hopkins, 2000). Based on this the $10 \mathrm{~m}$ sprint test procedure for the present study was reliable.

\section{Measures}

The dependent variables for the sprint time data were $5 \mathrm{~m}$ time, $10 \mathrm{~m}$ time and 5 to $10 \mathrm{~m}$ split time. All split times were displayed on an electronic hand held receiver and then transcribed to a Microsoft Excel file to be saved directly into coded individual participant folders.

\section{Statistical Analyses}

All statistical analyses were conducted using IBM SPSS Statistics (Version 22; SPSS, Inc, Chicago, IL, USA) and data are presented as pre to post baseline difference $\pm 95 \%$ confidence intervals. Time data were normally distributed for all groups at all time points, as assessed by the Shapiro-Wilk's test $(p>0.05)$. Repeated measures analyses of variance with one within-participant factor, i.e. time (baseline, $30 \mathrm{~s}, 2 \mathrm{~min}, 4 \mathrm{~min}, 6 \mathrm{~min}$ and $8 \mathrm{~min}$ ), and one between-participant factor, i.e. condition (C, 5R, 10R, 15R), were used. Significance was set at $p \leq 0.05$. The average of the three baseline trials was used for analysis. The Mauchly's test of sphericity was consulted and Greenhouse-Geisser correction was applied if sphericity was violated. Significant main effects of time were further investigated using pairwise comparisons relative to baseline with conservative Bonferroni confidence interval adjustment to the alpha level for the five comparisons made for each condition.

It had been previously noted that the optimal rest interval may differ for participants (Comyns et al., 2006). To determine if a particular volume of the gluteal activation warm-up protocol was beneficial to $10 \mathrm{~m}$ sprinting performance, the post test scores for each rest interval were examined and the scores that 
showed the greatest decrease in sprint time when compared to the baseline were selected for each participant in a manner similar to Bevan et al. (2010) and Comyns et al. (2006). A paired sample t-test was then performed on the baseline and fastest post intervention sprint data for the groups where the participants demonstrated faster post intervention $10 \mathrm{~m}$ times.

\section{Results}

Compared with the baseline times all groups had slower $5 \mathrm{~m}, 10 \mathrm{~m}$ and 5 to $10 \mathrm{~m}$ times after their specific intervention. These data are presented in Figures 1 to 3. Repeated measures ANOVA did reveal a significant main effect for 'time' (rest interval) for the $10 \mathrm{~m}$ time variable
$\left(\mathrm{F}_{3.65,131.46}=7.701, p \leq 0.001\right.$, partial $\left.\eta^{2}=0.176\right)$ and the 5 to $10 \mathrm{~m}$ split time variable $\left(\mathrm{F}_{5,180}=13.62, p \leq\right.$ 0.001 , partial $\left.\eta^{2}=0.274\right)$. The main effect for 'time' for the $5 \mathrm{~m}$ time variable was not significant $\left(\mathrm{F}_{3.59,129.18}=1.725, p=0.155\right.$, partial $\left.\eta^{2}=0.046\right)$. The simple main effect analysis for time showed that the $10 \mathrm{~m}$ time variable, relative to baseline, was significantly slower for the control group at the 6 min rest interval $(p=0.03)$ and the 8 min interval $(p=0.037)$. The $5 \mathrm{R}$ group revealed a significantly slower $10 \mathrm{~m}$ time at the $2 \mathrm{~min}$ rest interval $(p=$ 0.012). Similarly, the 5 to $10 \mathrm{~m}$ split time, relative to baseline, was significantly slower at the $30 \mathrm{~s}$ rest interval for the 10R group ( $p=0.024)$ and the $15 R$ group $(p=0.045)$.

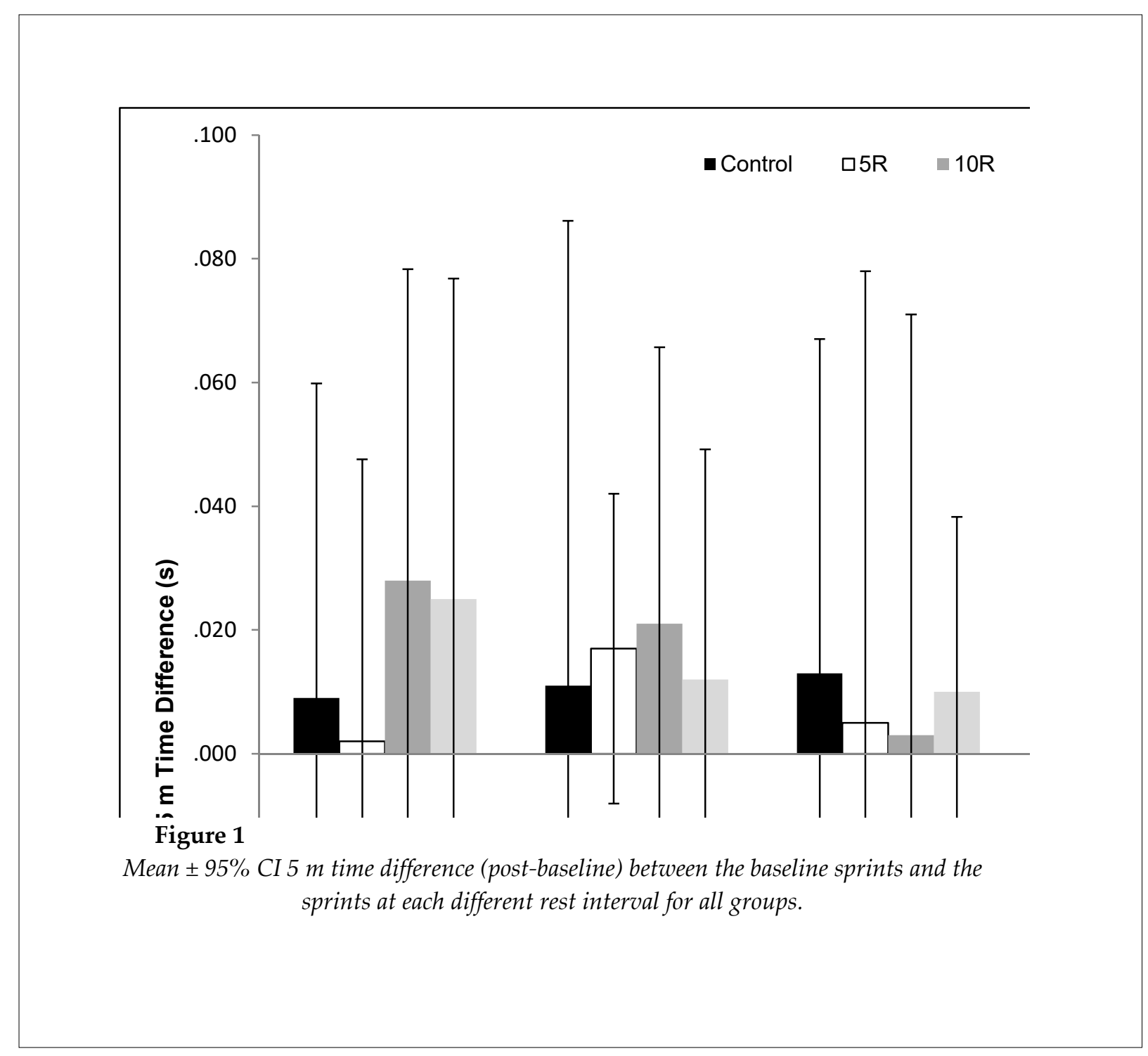




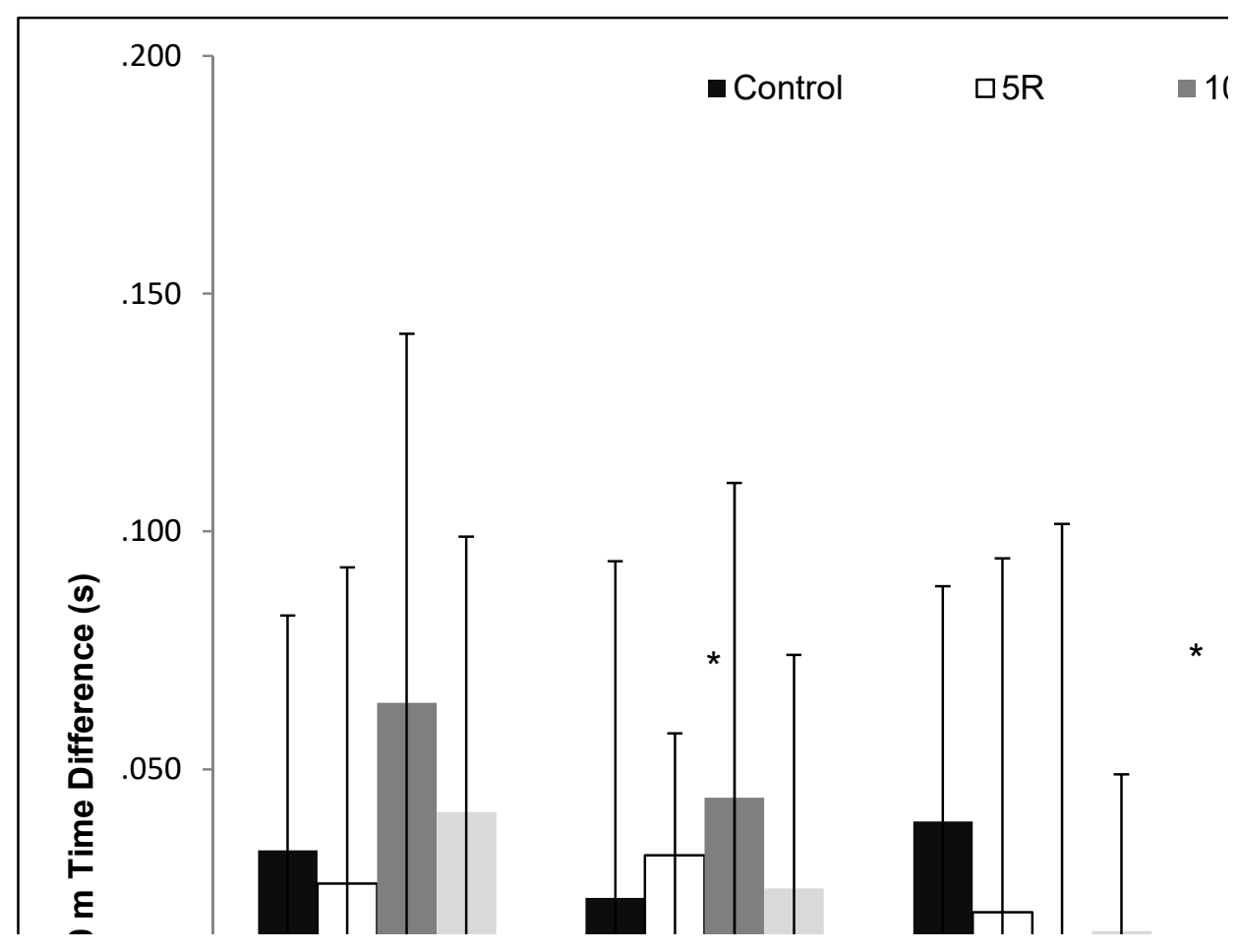

Figure 2

Mean $\pm 95 \%$ CI $10 \mathrm{~m}$ time difference (post-baseline) between the baseline sprints and the sprints at each different rest interval for all groups. ${ }^{*} p<0.05$

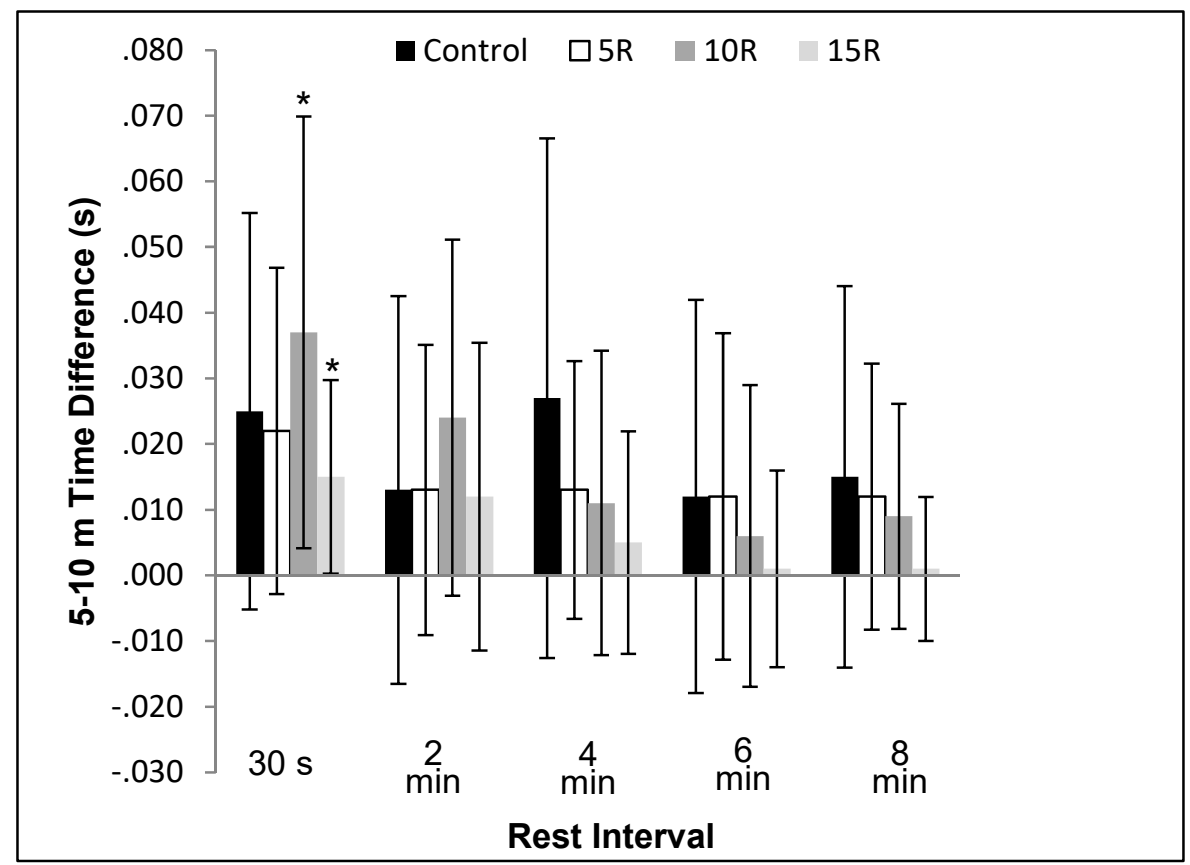

Figure 3

Mean $\pm 95 \%$ CI 5 to $10 \mathrm{~m}$ time difference (post-baseline) between the baseline sprints and the sprints at each different rest interval for all groups. ${ }^{*} p<0.05$ 


\begin{tabular}{|c|c|c|c|}
\hline \multicolumn{4}{|c|}{$\begin{array}{c}\text { Table } \mathbf{1} \\
\text { Gluteal Warm-Up Protocol Including EMG Muscle Activation Levels (adapted } \\
\text { from Crow et al., 2012). }\end{array}$} \\
\hline Exercise & As described by & $\begin{array}{c}\text { Gluteus Maximus } \\
\text { (\%MVIC) }\end{array}$ & $\begin{array}{c}\text { Gluteus Medius } \\
\text { (\%MVIC) }\end{array}$ \\
\hline Double Leg Bridge & Ekstrom et al. (2007) & $25 \pm 14$ & $28 \pm 17$ \\
\hline $\begin{array}{l}\text { Quadruped Lower Extremity } \\
\text { Lift }\end{array}$ & Ekstrom et al. (2007) & $42 \pm 17$ & $56 \pm 22$ \\
\hline Quadruped Hip Abduction & American Council on Exercise & N/A & N/A \\
\hline Side Lying Clam ( $60^{\circ}$ flexion) & Di Stefano et al. (2009) & $39 \pm 34$ & $38 \pm 29$ \\
\hline Side Lying Hip Abduction & Ekstrom et al. (2007) & $21 \pm 16$ & $39 \pm 17$ \\
\hline $\begin{array}{l}\text { Prone Single Leg Hip } \\
\text { Extension }\end{array}$ & Lewis and Sahrmann (2009) & $22 \pm 10$ & N/A \\
\hline Stability Ball Squat & American Council on Exercise & N/A & N/A \\
\hline
\end{tabular}

There was no statistically significant group $x$ time (rest interval) interaction in the $5 \mathrm{~m}$ time $\left(\mathrm{F}_{10.77,129.18}=0.943, p=0.5\right.$, partial $\left.\eta^{2}=0.073\right)$, $10 \mathrm{~m}$ time $\left(\mathrm{F}_{10.96,131.46}=1.454, p=0.157\right.$, partial $\eta^{2}=$ $0.108)$ and 5 to $10 \mathrm{~m}$ split time variables $\left(\mathrm{F}_{15,180}=\right.$ 1.495, $p=0.111$, partial $\eta^{2}=0.111$ ).

Regarding the analysis of the individual rest interval, $90 \%$ of the participants in the $15 \mathrm{R}$ group had a faster $10 \mathrm{~m}$ sprint time after their specific gluteal intervention. The rest interval where these faster $10 \mathrm{~m}$ times occurred differed for the participants in this group. Specifically, $33.3 \%$ of participants had their fastest times at the 8 minute interval while the remainder was evenly distributed across the 2, 4 and 6 minute rest intervals. The paired sample t-test that was performed on the baseline and fastest post intervention sprint data for this group revealed a significant improvement in the $10 \mathrm{~m}$ time (Baseline: $1.77 \pm 0.09 \mathrm{~s}$ vs. Best time: $1.75 \pm 0.10 \mathrm{~s}, p$ $=0.021)$.

\section{Discussion}

The results of this study provide insight into the effects of a low-load gluteal warm-up protocol on acceleration performance. Relative to baseline, the time to $5 \mathrm{~m}$ and $10 \mathrm{~m}$ and the 5 to 10 $\mathrm{m}$ split times were slower for all groups. The $5 \mathrm{R}$ group showed a significant increase in the $10 \mathrm{~m}$ time at the 2 minute rest interval. For the 5 to $10 \mathrm{~m}$ split time, the $10 \mathrm{R}$ and $15 \mathrm{R}$ groups had times at the $30 \mathrm{~s}$ interval that were significantly worse than their corresponding baseline times. These results would indicate that performing gluteal warm-up protocols with varying repetition ranges has a negative effect on post warm-up acceleration performance. This appears to contradict the findings of Comyns et al. (2015) and Crow et al. (2012).

Comyns et al. (2015) and Crow et al. (2012) reported that a low-load gluteal warm-up protocol had a PAP effect on subsequent squat jump (SJ) and CMJ performance, respectively. The results from the study by Comyns et al. (2015) indicated that a gluteal warm-up could enhance force production in SJs performed after 8 minute recovery. Crow et al. (2012) demonstrated that a low-load gluteal protocol was effective at acutely enhancing peak power output in the CMJ in elite athletes. Similar acute performance enhancing improvements do not appear evident in the current study. The precise mechanisms that underpin the post warm-up intervention activities, however, performed in both the Comyns et al. (2015) and the Crow et al. (2012) studies, are distinctly different to the mechanism underpinning the post intervention movement assessed in the current study. The CMJ used by Crow et al. (2012) is an example of a slow stretchshortening exercise (SSC). A slow SSC exercise involves contraction times in excess of $0.25 \mathrm{~s}$ and large angular displacements of the hips, knees 
and ankles (Schmidtbleicher, 1992). Comyns et al. (2015) reported PAP effects on SJ performance and the SJ involves no SSC mechanism. The present study used sprinting as the criterion activity and the fast SSC mechanism underpins this movement pattern. A fast SSC exercise is characterized by short contraction times $(<0.25 \mathrm{~s})$ and small angular displacements of the hips, knees and ankles (Schmidtbleicher, 1992). It has been hypothesized that the slow and fast SSC may represent different muscle action patterns that rely on differing biomechanical mechanisms, which can affect performance in different ways (Flanagan and Comyns, 2008). Therefore, PAP effects due to a low load gluteal warm-up on slow and non-SSC jump performance may not be evident in fast SSC performance.

Healy and Harrison (2014) researched the PAP effect of a low-load gluteal warm-up on drop jump (DJ) performance, which is a fast SSC movement. Neither a repeated measures ANOVA nor typical error analyses found positive performance enhancing effects on DJ performance as a result of the gluteal warm-up intervention. This is in support of the results reported in the current study. It could be hypothesized that drop jumps and sprinting are a fast SSC activity in which the fast rate of eccentric preload and short transition time between concentric and eccentric phases play a greater part in power production over the rate of force development of the gluteal, which would be seen in slower SSC activities such as the CMJ. Such a hypothesis would explain to a certain degree the lack of significant improvements in acceleration performance in the present study.

However, in support of previous studies, an individualized PAP effect was observed between participants. Previous research had indicated that the PAP response to a warm-up protocol was individual (Bevan et al., 2010; Comyns et al., 2006). Comyns et al. (2006) examined the effect of a 5 repetition maximum (5RM) back squat on CMJ performance at rest intervals of 30 seconds, 2, 4 and 6 minutes. Compared to the pre-test CMJs no significant differences were found in the post 5RM CMJ performance at any of the rest intervals $(p \geq 0.05)$. The authors reported that the improvement window was different for each participant and an analysis on the greatest increase in CMJ performance (flight time and peak ground reaction force) showed a significant increase in flight time for men and women. Similarly, Bevan et al. (2010) reported that the rest interval to elicit PAP effects due to a $3 R M$ back squat on sprinting performance was individual. Bevan et al. (2010) researched the PAP effect of a 3RM back squat on $10 \mathrm{~m}$ sprints that were performed 4, 8, 12 and 16 minutes post squat. No significant time effect with regard to $5 \mathrm{~m}$ and $10 \mathrm{~m}$ sprint times was reported $(p \geq 0.05)$. However, when individual responses to PAP were taken into account, a significant improvement in both $5 \mathrm{~m}$ and $10 \mathrm{~m}$ performance compared to the baseline sprints was observed. The present results support the findings of Bevan et al. (2010) and Comyns et al. (2006). The improved $10 \mathrm{~m}$ times reported for the 15R group occurred at different rest intervals and the analysis indicated that these grouped improvements were statistically significant. The majority of the current participants $(33.3 \%)$ performed their fastest $10 \mathrm{~m}$ sprint at the 8 minute interval and this finding replicates those previously found by Bevan et al. (2010) and Turner et al. (2015) when sprinting was the criterion activity. When compared to the baseline sprints, none of the participants in the $15 \mathrm{R}$ group had a faster post warm-up $10 \mathrm{~m}$ time at the 30 second rest interval. Such findings would seem to indicate that a low-load 15 repetition gluteal warm-up protocol has a potentiating effect on acceleration performance provided that the rest interval is adequately and individually determined. Future research should examine longer rest intervals after the gluteal warm-up protocols and consider using a typical error analysis method, similar to Healy and Harrison (2014), to assess individual PAP responses to gluteal activation protocols.

\section{Conclusion}

The results of the present study suggest that there is no specific rest interval that causes a PAP effect on acceleration performance due to a low-load gluteal warm-up at different repetition ranges. Neither the $5 R, 10 R$ or $15 R$ group demonstrated improved sprint times at the various rest intervals after their specific gluteal warm-up. These times were all slower and such a finding would suggest that there is no specific rest interval that results in a PAP effect on acceleration 
performance as a result of a gluteal activation warm-up.

However, the participants in the $15 \mathrm{R}$ group elicited faster $10 \mathrm{~m}$ sprint times after the 15 repetition gluteal warm-up. These times were run at different rest intervals and the significant findings here would suggest that a 15 repetition gluteal warm-up protocol has a performance enhancing effect on $10 \mathrm{~m}$ sprinting provided the rest interval is individually determined. Therefore, practitioners should consider using such a warm-up protocol which requires little equipment and can be easily incorporated into training and competition routines, with individually assessed rest intervals to enhance sprinting performance of rugby union players.

\section{References}

Atkinson G, Reilly T. Circadian variation in sports performance. Sports Med, 1996; 21: $292-312$

Baker D, Nance S. The relation between running speed and measures of strength and power in professional rugby league players. J Strength Cond Res, 1999; 13: 230-235

Bevan H, Cunningham D, Tooley E, Owen N, Cook C, Kilduff L. Influence of postactivation potentiation on sprinting performance in professional rugby players. J Strength Cond Res, 2010; 24: 1-5

Chatzopoulos D. Postactivation potentiation effects after heavy resistance exercise on running speed. $J$ Strength Cond Res, 2007; 21: 1278-1281

Comyns T, Harrison AJ, Hennessy L. Effect of squatting on sprinting performance and repeated exposure to complex training in male rugby players. J Strength Cond Res, 2010; 24: 610-618

Comyns TM, Harrison AJ, Hennessy LK, Jensen RL. The optimal complex training rest interval for athletes from anaerobic sports. J Strength Cond Res, 2006; 20: 471-476

Comyns T, Kenny I, Scales G. Effects of a low load gluteal warm-up on explosive jumping performance. $J$ Hum Kinet, 2015; 46: 177-187

Crow JF, Buttifant D, Kearny SG, Hrysomallis C. Low load exercises targeting the gluteal muscle group acutely enhance explosive power output in elite athletes. J Strength Cond Res, 2012; 26(2): 438-442

Earp J, Newton R. Advances in electronic timing systems: Considerations for selecting an appropriate timing system. J Strength Cond Res, 2012; 26: 1245-1248

Flanagan EP, Comyns TM. The use of contact time and reactive strength index to optimize fast stretchshortening cycle training. J Strength Cond Res, 2008; 21: 67-72

Healy R, Harrison AJ. The effects of a unilateral gluteal activation protocol on single leg drop jump performance. Sports Biomech, 2014; 13(1): 33-46

Hodgson M, Docherty D, Robbins D. Post-activation potentiation. Sports Med, 2005; 35: 585-595

Hopkins WG. Measures of Reliability in Sports Medicine and Science. Sports Med, 2000; 30(1): 1-15

Jeffreys I. Warm up revisited - the 'ramp' method of optimising performance preparation. UKSCA Journal, 2006; 6: 15-19

Kyrolainen H, Avela J, Komi PV. Changes in muscle activity with increasing running speed. J Sport Sci, 2005; 23: 1101-1109

Little T, Williams A. Effects of differential stretching protocols during warm-ups on high speed motor capacities in professional soccer players. J Strength Cond Res, 2006; 20: 203-207

Matthews M, Comfort P, Crebin R. Complex training in ice hockey: the effects of a heavy resisted sprint on subsequent ice-hockey sprint performance. J Strength Cond Res, 2010; 24: 2883-2887

McBride JM, Nimphius S, Erickson TM. The acute effects of heavy-load squats and loaded countermovement jumps on sprint performance. J Strength Cond Res, 2005; 19: 893-897

Nagano A, Komura T, Fukashiro S, Himeno R. Force, work and power output of lower limb muscles during human maximal-effort countermovement jumping. J Electromyogr Kines, 2005; 15: 367-376 
Novacheck T. The biomechanics of running. Gait and Posture, 1998; 7: 77-95

Palastanga N, Field D, Soames R. Anatomy and human movement. $4^{\text {th }} e d$. Edinburgh: Butterworth Heinemann; 2002

Schmidtbleicher D. Training for power events. In: Komi PV. The Encyclopedia of Sports Medicine. Vol 3: Strength and Power in Sport. Oxford: Blackwell, 169-179; 1992

Turner AP, Bellhouse S, Kilduff LP, Russell M. Postactivation potentiation of sprint acceleration performance using plyometric exercise. J Strength Cond Res, 2015; 29: 343-350

Weimann K, Tidow G. Relative activity of hip and knee extensors in sprinting - implications for training. New Studies in Athletics, 1995; 10: 29-49

Whelan N, O'Regan C, Harrison AJ. Resisted sprints do not acutely enhance sprinting performance. J Strength Cond Res, 2014; 28: 1858-1866

\section{Corresponding author:}

\section{Thomas Comyns}

Biomechanics Research Unit

Department of Physical Education and Sport Sciences

University of Limerick

Limerick, Ireland

Phone: +35361234738

E-mail: tom.comyns@ul.ie 